\title{
The analysis of TM-mode and TE-mode optical responses of metallic nanostructures by new surface integral equations
}

\author{
J.-W. Liaw \\ Department of Mechanical Engineering, Chang Gung University, \\ Kwei-Shan, Tao-Yuan, Taiwan
}

\begin{abstract}
Two sets of new surface integral equations, based on the Stratton-Chu formulation, are developed to analyze the interactions of an incident electromagnetic wave with a 2D metallic nanostructure for the transverse-magnetic (TM) mode and the transverse-electric (TE) mode, respectively. For the former, the surface integral equations are in terms of the surface components of the tangential magnetic field $H_{z}$, the normal displacement field $D_{n}$, and the tangential electric field $E_{t}$. As to the latter, the equations are in terms of the surface components of the tangential electric field $E_{z}$, the normal magnetic flux density $B_{n}$, and the tangential magnetic field $H_{t}$. The numerical results show that for TM mode a standing wave pattern is observed on the backside of a metallic nanoscatterer with a size of several hundreds nanometers, which is caused by two surface plasmon waves creeping along the boundary clockwise and counterclockwise. However there is only a shadow zone on the backside of the metallic scatterer for TE mode.

Keywords: surface plasmon resonance, surface plasmon wave, metallic nanostructure, transverse magnetic mode, transverse electric mode, surface integral equations.
\end{abstract}

\section{Introduction}

Recently a new topic-plasmonics attracts much attention in nanooptics [1-4]. It is concerned with the interaction of light with metallic nanostructures. Because of the free electrons in metals, the permittivity of metal (e.g. Au and Ag) is not 
only frequency-dependent but also a complex number with a negative real part and a positive imaginary part in certain range of VU to NIR. When a nanometersized metallic scatterer is illuminated by an incident light at a specific frequency, this highly dispersive property makes them exhibit a collective motion of electrons in the metal to induce a strong scattering and absorption of the illuminating light. The phenomenon is the so-called surface plasmon resonance (SPR). The main difference of the SPR of a metallic nanoscatterer caused by an electromagnetic (EM) wave from the regular resonance of the acoustic or elastic waves problems is that the size of the metallic nanoscatterer is much smaller than the wavelength of the incident light. In addition, the frequency of SPR depends on the metal's size, shape, and the permittivity of the surrounding medium sensitively. For a 2D metallic nanostructure (the length in z-direction is infinitely long), the SPR can be induced only by an EM wave of p-polarization (the polarization of the electric field is in-plane), but not by that of $s$-polarization (the polarization of the electric field is anti-plane). The unique behaviors of a nanometer-sized metallic scatterer at SPR are the significantly large scattering and absorption cross sections at the far field, and the strong electric field at near field. However, when the size of the metallic nanoscatterer becomes larger, the property of the frequency-selected SPR gradually disappears, and a surface plasmon wave gradually forms on the surface of the metal for a TM-mode problem only. Since the behaviors of a nanometer-sized scatterer (e.g. several tens nanometers) are intensively investigated in lots of papers [1-4], we focus on the studies of a 2D metallic nanoscatterer with a size of several hundreds nanometers in this paper to demonstrate the difference of the TM-mode and TEmode responses. In this paper, two sets of surface integral equations derived from the Stratton-Chu formulation [5] are used and implemented by BEM to simulate the interactions of an EM wave in TM-mode and in TE-mode with a 2D metallic nanoscatterer, respectively.

\section{Theory}

In this paper, the time harmonic responses of an EM plane wave propagating in a host (exterior) to interact with a single metallic scatterer (interior) are considered; the time harmonic factor is $\exp (-\mathrm{i} \omega t)$, where $\mathrm{i}=\sqrt{-1}$. The permittivity of each material is denoted by $\varepsilon_{j}$, and the permeability by $\mu_{j}$, where $j=1$ is for the host, and $j=2$ for the scatterer.

\subsection{TM mode}

For a 2D TM-mode ( $p$-polarization) problem, the electric field is in plane and can be expressed as $\mathbf{E}=E_{x} \mathbf{e}_{\mathbf{x}}+E_{y} \mathbf{e}_{\mathbf{y}}$, and the magnetic field is $\mathbf{H}=H_{z} \mathbf{e}_{\mathbf{z}}$. Here, $\mathbf{e}_{\mathbf{x}}, \mathbf{e}_{\mathbf{y}}$ and $\mathbf{e}_{\mathrm{z}}$ are the unit vectors of $\mathrm{x}, \mathrm{y}$ and $\mathrm{z}$ directions, respectively. The total fields in the exterior domain can be decomposed of two parts; one is the incident field, and the other the scattering field, i.e. $\mathbf{E}=\mathbf{E}^{(i)}+\mathbf{E}^{(s)}$ and $H_{z}=H_{z}^{(i)}+H_{z}^{(s)}$. Throughout 
the paper, the superscript ' $(i)$ ' represents the incident field, and ' $(s)$ ' the scattering field. A set of surface integral equations can be derived from the Stratton-Chu formulation [5] as

$$
\begin{aligned}
& H_{z}(\mathbf{x})=H_{z}^{(i)}(\mathbf{x})-\int_{S} H_{z}\left(\mathbf{x}^{\prime}\right) \mathbf{n}^{\prime} \cdot \nabla^{\prime}\left[G_{1}-G_{2}\right] d l^{\prime}-i \omega \int_{S} E_{t}\left(\mathbf{x}^{\prime}\right)\left[\varepsilon_{1} G_{1}-\varepsilon_{2} G_{2}\right] d l^{\prime}, \\
& \mathbf{x} \in S_{12} \\
& D_{n}(\mathbf{x})=D_{z}^{(i)}-i \omega \int_{S_{12}} H_{z}\left[\varepsilon_{1} \mu_{1} G_{1}-\varepsilon_{2} \mu_{2} G_{2}\right] \mathbf{n} \cdot d \mathbf{l}^{\prime}-\int_{S_{12}} D_{n} \mathbf{n} \cdot \nabla^{\prime}\left[G_{1}-G_{2}\right] d l^{\prime}+ \\
& \int_{S_{12}} E_{t} \mathbf{n} \cdot \mathbf{e}_{z} \times \nabla^{\prime}\left[\varepsilon_{1} G_{1}-\varepsilon_{2} G_{2}\right] d l^{\prime}, \mathbf{x} \in S_{12} \\
& E_{t}(\mathbf{x})=E_{t}^{(i)}-i \omega \int_{S_{12}} H_{z}\left[\mu_{1} G_{1}-\mu_{2} G_{2}\right] \mathbf{t} \cdot d \mathbf{l}^{\prime}-\int_{S_{12}} D_{n} \mathbf{t} \cdot \nabla^{\prime}\left[\frac{G_{1}}{\varepsilon_{1}}-\frac{G_{2}}{\varepsilon_{2}}\right] d l^{\prime}+ \\
& \quad E_{S_{12}} E_{t} \mathbf{t} \cdot \mathbf{e}_{z} \times \nabla^{\prime}\left[G_{1}-G_{2}\right] d l^{\prime}, \mathbf{x} \in S_{12}
\end{aligned}
$$

where $S_{12}$ is the interface of the host and the scatterer, the unit normal vector $\mathbf{n}$ of $S_{12}$ is in the inner direction, and the unit tangential vector is defined as $\mathbf{t}=\mathbf{n} \times \mathbf{e}_{\mathbf{z}}$. These surface integral equations [2] are in terms of the surface components of the tangential magnetic field $H_{z}$, the normal displacement field $D_{n}$, and the tangential electric field $E_{t}$. Here, the Green functions $G_{j}$ of medium $j, j=1,2$, are written as

$$
G_{j}\left(\mathbf{x}, \mathbf{x}^{\prime}\right)=\frac{\mathrm{i}}{4} H_{0}^{(1)}\left(k_{j} r\right)
$$

where $r$ is the distance $r=\left|\mathbf{x}-\mathbf{x}^{\prime}\right|$, and the wavenumbers are $k_{j}=\omega \sqrt{\varepsilon_{j} \mu_{j}}$.

\subsection{TE mode}

For a 2D TE-mode ( $s$-polarization) problem, the magnetic field is in plane and can be expressed as $\mathbf{H}=H_{x} \mathbf{e}_{\mathbf{x}}+H_{y} \mathbf{e}_{\mathbf{y}}$, and the electric field is $\mathbf{E}=E_{z} \mathbf{e}_{\mathbf{z}}$. The total fields in the exterior domain can be decomposed of two parts; one is the incident field, and the other the scattering field, i.e. $\mathbf{H}=\mathbf{H}^{i}+\mathbf{H}^{s}$ and $E_{z}=E_{z}{ }^{i}+E_{z}{ }^{s}$. The surface integral equations derived from the Stratton-Chu formulation can be written in terms of the surface components of the tangential electric field $E_{z}$, the normal magnetic flux density $B_{n}$, and the tangential magnetic field $H_{t}$ as,

$$
\begin{aligned}
& E_{z}(\mathbf{x})=E_{z}^{(i)}-\int_{S_{12}} E_{z}\left(\mathbf{x}^{\prime}\right) \mathbf{n}^{\prime} \cdot \nabla^{\prime}\left[G_{1}-G_{2}\right] d l^{\prime}+i \omega \int_{S_{12}} H_{t}\left(\mathbf{x}^{\prime}\right)\left[\mu_{1} G_{1}-\mu_{2} G_{2}\right] d l^{\prime} \\
& , \mathbf{x} \in S_{12}
\end{aligned}
$$




$$
\begin{aligned}
& B_{n}(\mathbf{x})=B_{n}^{(i)}+i \omega \int_{S_{12}} E_{z}\left[\varepsilon_{1} \mu_{1} G_{1}-\varepsilon_{2} \mu_{2} G_{2}\right] \mathbf{n} \cdot d \mathbf{l}^{\prime}-\int_{S_{12}} B_{n} \mathbf{n} \cdot \nabla^{\prime}\left[G_{1}-G_{2}\right] d l^{\prime}+ \\
& \int_{S_{12}} H_{t} \mathbf{n} \cdot \mathbf{e}_{z} \times \nabla^{\prime}\left[\mu_{1} G_{1}-\mu_{2} G_{2}\right] d l^{\prime}, \mathbf{x} \in S_{12} \\
& H_{t}(\mathbf{x})=H_{t}^{(i)}+i \omega \int_{S_{12}} E_{z}\left[\varepsilon_{1} G_{1}-\varepsilon_{2} G_{2}\right] \mathbf{t} \cdot d \mathbf{l}^{\prime}-\int_{S_{12}} B_{n} \mathbf{t} \cdot \nabla^{\prime}\left[\frac{G_{1}}{\mu_{1}}-\frac{G_{2}}{\mu_{2}}\right] d l^{\prime}+ \\
& \int_{S_{12}} H_{t} \mathbf{t} \cdot \mathbf{e}_{z} \times \nabla^{\prime}\left[G_{1}-G_{2}\right] d l^{\prime}, \mathbf{x} \in S_{12}
\end{aligned}
$$

\section{Numerical results and discussion}

Consider a 2D scatterer of an infinite-long circular cylinder which is embedded in air. If an incident EM plane wave is in TM-mode (the polarization of the electric field is in-plane) to impinge upon the scatterer, Eqns. (1) to (3) are used to calculate the surface components along the boundary, and then the corresponding integral representations [2] are used to obtain the field values in the exterior and interior domains. Take a typical case as an example; an incident wave of TM-mode propagates from the left to the right hand side at $\omega=4.558 \times 10^{15} \mathrm{rad} / \mathrm{s}(3 \mathrm{eV})$ to impinge upon a silver cylinder $(r=200 \mathrm{~nm})$. The distributions of the amplitude of the total electric field and the total magnetic field in the near field of the scatterer are depicted in Fig. 1(a) and 1(b), where $\varepsilon_{l r}=1$ and $\varepsilon_{2 r}=(-4.422171,0.73006)$ [6]. For this case, the wavelength of the incident wave is $\lambda=413.3 \mathrm{~nm}$. The numerical results show an interference pattern on the backside of the metallic scatterer. This is because when the illuminating light impinges upon the silver scatterer, a part of the photon's energy is converted into two surface plasmon waves creeping along the circumference of the silver cylinder counterclockwise and clockwise, respectively. The two opposite-directional surface plasmon waves will interfere with each other to induce a standing wave on the backside of the scatterer.

If the incident EM plane wave is in TE mode (the polarization of the electric field is anti-plane), Eqns. (5) to (7) are used for calculation. Compared to the TM-mode, there is only a shadow zone, rather than a standing wave, to be observed on the backside of the metallic scatterer for TE-mode as depicted in Fig. 2(a) and 2(b). The phenomenon of shadow zone is consistent with the result of a perfect-conductor scatterer. In addition, due to the shielding effect of metals on the EM field, the EM field cannot directly transmit through the metallic scatterer, except within a thin skin depth, for both TM and TE modes, as shown in Figs. 1 and 2. Since the surface plasmon wave exists only in TM mode [2], the scattering responses of an EM wave in the near field of a metallic nanoscatterer are totally different in the TM and TE modes. 

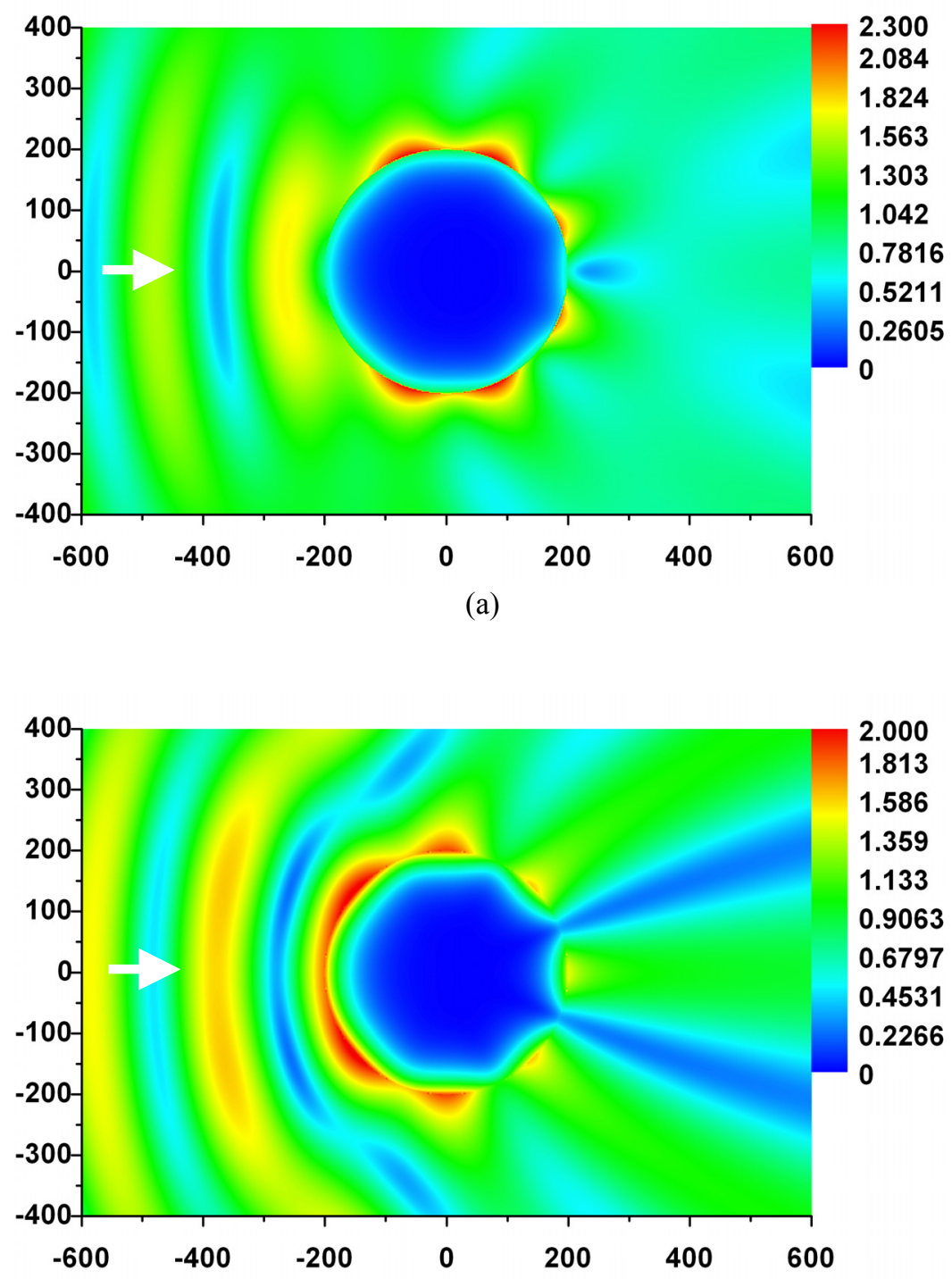

(b)

Figure 1: (a) The total electric field distribution in the vicinity of a silver cylinder with $r=200 \mathrm{~nm}$ irradiated by a TM-mode plane wave at $\omega=4.558 \times 10^{15} \mathrm{rad} / \mathrm{s}(3 \mathrm{eV})$, where $\varepsilon_{1 r}=1, \varepsilon_{2 r}=(-4.422171,0.73006)$. (b) The total magnetic field distribution. 

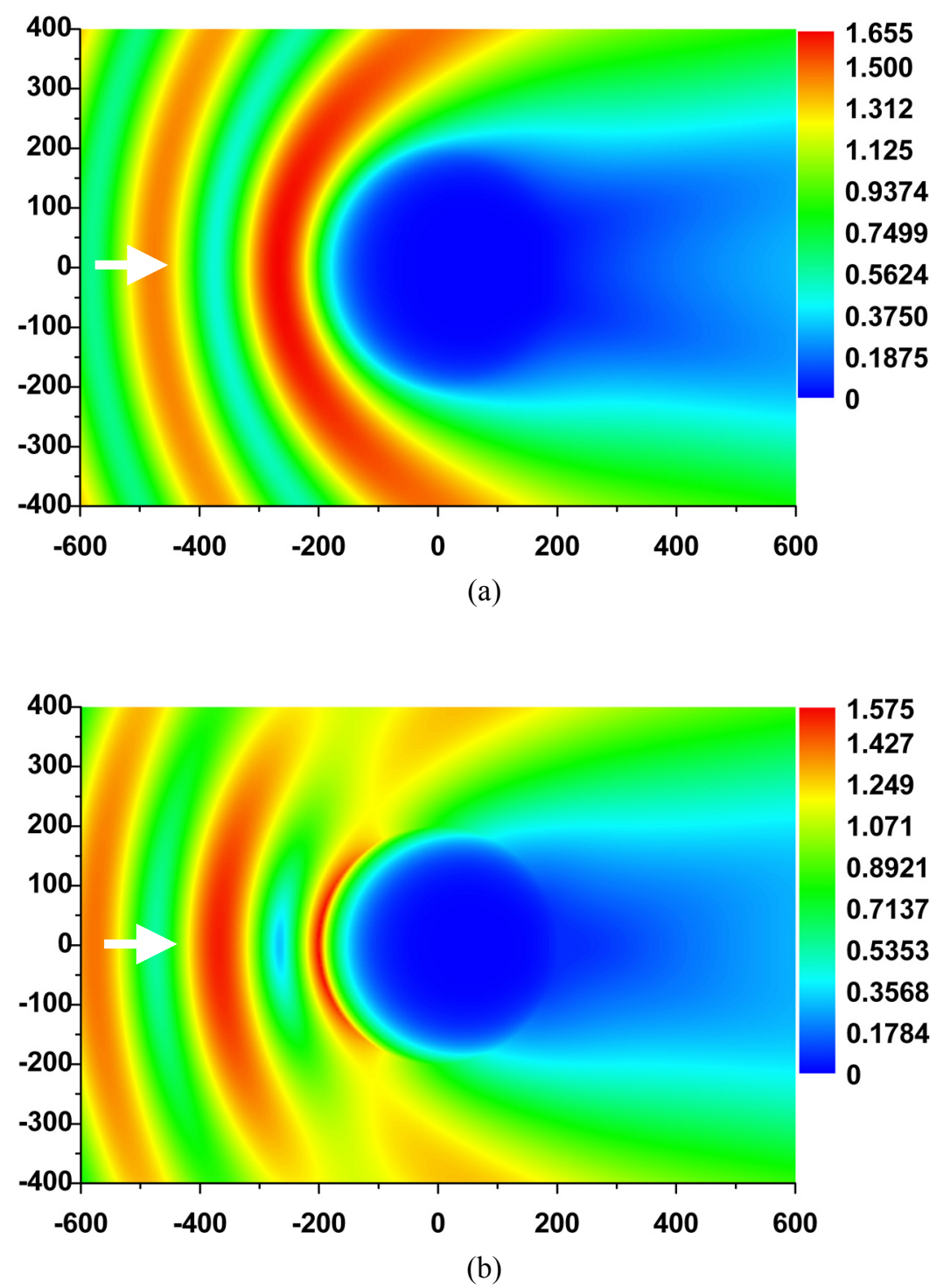

Figure 2: The total electric field distribution in the vicinity of a silver cylinder with $r=200 \mathrm{~nm}$ irradiated by a TE-mode plane wave at $\omega=4.558 \times 10^{15} \mathrm{rad} / \mathrm{s}(3 \mathrm{eV})$, where $\varepsilon_{l r}=1, \varepsilon_{2 r}=(-4.422171,0.73006)$. (b) The total magnetic field distribution. 


\section{References}

[1] Kottmann, J.P., Martin, J.F., Smith, D. R., Schultz, S., Spectral response of plasmon resonant nanoparticles with a non-regular shape, Opt. Express 6, pp. 213-219, 2000.

[2] Liaw, J.-W., Simulation of surface plasmon resonance of metallic nanoparticles by boundary-element method, J. Opt. Soc. Am., A 23(1), pp. 108-116, 2006.

[3] Liaw, J.-W., Analysis of the surface plasmon resonance of a single coreshelled nanocomposite by surface integral equations, Eng. Anal. with Boundary Elements, 30(9), pp. 734-745, 2006.

[4] Liaw, J.-W., New surface integral equations for the light scattering of multi metallic nanoscatterers, Eng. Anal. with Boundary Elements 31(4), pp. 299-310, 2007.

[5] Stratton, A., Electromagnetic theory (McGraw-Hill, New York, 1941), pp. 464-467.

[6] Palik, E. D., Handbook of optical constants of solids (Academic Press, New York, 1985) 